\title{
Mantenimiento y adaptación cultural de diferentes grupos inmigrantes: variables predictoras
}

\author{
Marisol Navas *, Lucía López-Rodríguez e Isabel Cuadrado \\ Universidad de Almería, España
}

\begin{abstract}
Resumen: Este estudio pretende analizar las dimensiones de mantenimiento y adaptación cultural expresadas por inmigrantes de diferentes orígenes en España, así como las variables psicosociales y sociodemográficas que las predicen. Se realizó un estudio correlacional en el que participaron inmigrantes marroquíes $(N=71)$, rumanos $(N=74)$ y ecuatorianos $(N=70)$ que respondieron a un cuestionario manifestando el grado de mantenimiento de su cultura de origen, de adaptación a la cultura de acogida, y de obligación percibida a adaptarse a la cultura de acogida. También se midieron los estereotipos sobre los españoles (dimensiones de calidez y competencia), la amenaza exogrupal, la identificación con el endogrupo, la similitud intergrupal percibida, la naturaleza del contacto con el exogrupo, la percepción de la relación intergrupal, el enriquecimiento percibido debido a la inmigración, y algunas variables sociodemográficas. Los resultados mostraron la importancia del origen de los inmigrantes en todas las variables del estudio. Además, los predictores de las dimensiones de aculturación también eran diferentes dependiendo del grupo inmigrante considerado. Estos resultados enfatizan la necesidad de intervenciones específicas, ya que cada grupo inmigrante afronta el proceso de adaptación a una nueva sociedad de acogida de forma diferente.

Palabras clave: Mantenimiento cultural; adaptación cultural; inmigrantes;
\end{abstract} efecto del origen nacional; variables predictoras.

\section{Introducción}

El proceso migratorio es un acontecimiento de tal complejidad y diversidad que cada persona lo experimenta y lo afronta de forma diferente. La cultura y la historia del país de origen, la experiencia personal, las razones por las que se decide emigrar y multitud de otras variables (psicosociales, sociodemográficas, etc.) pueden influir profundamente en la forma de afrontar este proceso en el país de acogida, así como en el tipo y la naturaleza de las relaciones que se establecen con el grupo mayoritario.

En efecto, cuando las personas se ven envueltas en un proceso migratorio, inevitablemente entran en contacto con una cultura diferente a la propia, y esto desencadena determinados cambios tanto en los inmigrantes, como en la sociedad de acogida, lo que es conocido generalmente como "proceso de aculturación" (Redfield, Linton y Herskovits, 1936).

Los diferentes modelos de aculturación psicológica reconocen el papel fundamental del origen de los inmigrantes sobre la forma en que se desarrolla su proceso de aculturación y adaptación en una nueva sociedad de acogida (p.e., Berry, 1997; Bourhis, Moïse, Perreault y Senécal, 1997; Bourhis, Montaruli, El-Geledi, Harvey y Barrette, 2010; Navas et al., 2004; Navas y Rojas, 2010). La razón fundamental es que estas diferencias en origen también se acompañan de

* Dirección para correspondencia [Correspondence address]: Marisol Navas Luque. Departamento de Ciencias Humanas y Sociales. Universidad de Almería, Facultad de Psicología. Cañada de San Urbano s/n.04120. Almería (España).E-mail:msnavas@ual.es
Title: Cultural maintenance and adaptation of different immigrant groups: Predictor variables.

Abstract: This study aimed to analyze the cultural maintenance and adaptation dimensions expressed by Moroccan, Romanian, and Ecuadorian immigrants in Spain, as well as psychosocial and social-demographic variables that predicted such tendencies. A correlational study was carried out with immigrants of Moroccan $(N=71)$, Romanian $(N=74)$, and Ecuadorian origin $(N=70)$ who responded to a questionnaire reporting their tendency to maintain the original culture, to adapt to the host culture, and their perceived duty to adapt to the host culture. It was also measured the stereotype dimensions of warmth and competence about Spanish people, out-group threat, in-group identification, perceived in-out-group similarity, nature of out-group contact, perception of inter-group relations, perceived enrichment by immigration, and some social-demographic variables. Results showed the importance of immigrants' origin on all variables of the study. In addition, the predictors of acculturation dimensions were different depending on the group considered. These results highlight the need for specific interventions, as each immigrant group faces the adaptation process to the new host society in a different way.

Key words: Cultural maintenance; cultural adaptation; immigrants; national origin effect; predictor variables.

diferencias en una serie de características (p.e., sociodemográficas, económicas, psicosociales, culturales) que, sin duda, influirán en estos procesos.

¿Por qué es tan importante el origen de los inmigrantes en la forma y el resultado de afrontar su proceso migratorio en la nueva sociedad? Parece lógico suponer que el proceso de adaptación que debe producirse al llegar a una nueva sociedad de acogida se realice desde la perspectiva de la propia cultura (los propios valores, normas, patrones culturales e identidades), pero teniendo en cuenta también las diferentes expectativas y estereotipos que la sociedad de acogida mantiene sobre diferentes grupos inmigrantes (Worchel, 2004). Además, los inmigrantes no se despojan sin más de sus valores y costumbres de origen o los cambian por otros nuevos de forma automática o similar en todos los casos, sino que, dependiendo de sus patrones culturales (y de lo que la sociedad de acogida les permita), llevan a cabo un proceso de selección y modificación específica para adaptarse a su nuevo ambiente (Buriel, 1993; Mendoza, 1989). Por eso este proceso es diferente dependiendo de su origen nacional o étnico.

Las investigaciones realizadas desde distintos modelos de aculturación, en diferentes contextos culturales y con diversos grupos inmigrantes, apoyan la idea de que las estrategias/actitudes o dimensiones de aculturación (i.e., mantenimiento de la cultura de origen y adopción de la cultura de acogida) de los inmigrantes varían dependiendo de su origen étnico o nacional (véase, p.e., Bourhis, Barrette, El-Geledi y Schmidt, 2009; Choi y Thomas, 2009; Piontkowski, Florack, Hoelker y Obdrzálek, 2000; Schmitz, 1987). También los estudios realizados en España aplicando el Modelo Ampliado de Aculturación Relativa (MAAR) muestran la importancia 
del origen de los inmigrantes sobre estas dimensiones (mantenimiento y/o adopción cultural). Así, los inmigrantes magrebíes (Navas et al., 2004) y los de origen rumano (Navas y Rojas, 2010), prefieren mantener y adoptar en igual medida, mientras que los procedentes del África subsahariana están dispuestos a mantener menos y adoptar más (Navas et al., 2004), y los procedentes de Ecuador prefieren lo contrario: más mantenimiento de costumbres de origen (Navas y Rojas, 2010).

El origen de los inmigrantes está relacionado también con otras variables psicosociales, como la similitud intergrupal percibida, el contacto con el exogrupo, el grado de identificación endogrupal o la amenaza exogrupal percibida, variables que, a su vez, están estrechamente relacionadas con el proceso de adaptación de los inmigrantes a una nueva sociedad de acogida. Así, Rohmann, Florack y Piontkowski (2006) muestran que, en Alemania, los inmigrantes turcos, en comparación con los italianos, percibían menos similitud entre su grupo y los alemanes, más amenaza por parte de la sociedad de acogida y mayor discordancia en el contacto con alemanes (i.e., los inmigrantes desean mantener contacto con el exogrupo, pero creen que éste no es deseado por los miembros del exogrupo).

En España, los estudios realizados con el MAAR también han mostrado diferencias en distintas variables psicosociales en diferentes grupos inmigrantes. Así, los inmigrantes de origen magrebí, en comparación con los procedentes del África subsahariana, se perciben más similares a los españoles en diferentes aspectos, aunque no difieren en su grado de contacto (Navas et al., 2004). Por su parte, los inmigrantes procedentes de Rumanía, en comparación con los de origen ecuatoriano, se perciben también más similares a los españoles y consideran que el contacto con éstos ha sido más positivo (Navas y Rojas, 2010).

Además de estas variables psicosociales tradicionalmente estudiadas en las relaciones autóctonos-inmigrantes, en los últimos años se ha empezado a reconocer el papel que otras variables psicosociales relevantes podrían tener sobre estos procesos. Una de ellas es el contenido de los estereotipos mantenidos sobre el exogrupo. En este sentido, desde el modelo de contenido de los estereotipos (Stereotype Content Model, SCM; Cuddy, Fiske y Glick, 2008; Fiske, Cuddy, Glick y Xu, 2002; Fiske, Xu, Cuddy y Glick, 1999) se considera que la percepción que tenemos sobre otros grupos sociales se elabora a partir de dos dimensiones básicas de estereotipos: la calidez y la competencia. La dimensión de calidez nos ayuda a anticipar las intenciones de los otros hacia nosotros, y comprende rasgos como dignos de confianza, sinceros, amistosos, bien intencionados, afectuosos y buenas personas. La dimensión de competencia, por su parte, nos ayuda a conocer la capacidad de los otros para lograr sus intenciones u objetivos, y comprende rasgos como capaces, competentes, eficientes, cualificados, seguros de sí mismos e inteligentes.

Los escasos trabajos realizados hasta el momento con esta variable en relación con el proceso de aculturación se han focalizado en la percepción estereotipada que la población mayoritaria mantiene sobre los diferentes grupos inmigrantes (p.e., Maisonneuve y Testé, 2007; Montreuil y Bourhis, 2001), pero no al contrario. Los resultados de estas investigaciones muestran que los estereotipos que se mantienen sobre los grupos minoritarios condicionan la preferencia de los autóctonos por el mantenimiento y/o la adopción cultural de los inmigrantes y que, a la vez, esto tendría consecuencias sobre las preferencias de los propios inmigrantes. Así pues, parece totalmente justificado investigar también la percepción estereotipada que los inmigrantes tienen sobre los autóctonos y analizar su relación con sus preferencias sobre estas dos dimensiones. Basándonos en los estudios mencionados, podría esperase que las percepciones estereotipadas de los inmigrantes sobre la sociedad de acogida (i.e., percibir a los autóctonos en mayor o menor medida como cálidos y/o competentes) pudieran estar relacionadas con la forma en que afrontan su proceso de adaptación en esta sociedad. De este modo, las dos dimensiones generales de estereotipos propuestas desde el modelo de contenido de los estereotipos (calidez y competencia) podrían llegar a funcionar como catalizadoras de las relaciones intergrupales, tal y como anticiparon Lee y Fiske (2006).

Finalmente, el origen de los inmigrantes también está relacionado con las variables que predicen las estrategias/actitudes y dimensiones de aculturación, y que serán diferentes para cada grupo inmigrante. Así, existen numerosas variables de carácter individual, grupal, intergrupal y contextual que parecen condicionar las preferencias de los inmigrantes por unas u otras estrategias/actitudes o dimensiones de aculturación. Este resultado es una constante en los estudios mencionados anteriormente, realizados en distintos países y con diferentes grupos inmigrantes (véase, p.e., Choi y Thomas, 2009; Navas et al., 2004; Navas y Rojas, 2010; Piontkwoski et al., 2000), si bien las variables predictoras incluidas en cada estudio son diferentes y no se han abordado conjuntamente en un único trabajo.

\section{El presente estudio}

Basándonos en la literatura previa, el presente trabajo tiene como objetivo último profundizar en la comprensión de los factores que influyen en cómo los inmigrantes afrontan su proceso migratorio en el país de acogida, así como resaltar la especificidad de este proceso para diferentes grupos inmigrantes en función de su procedencia. Es evidente que el conocimiento de las variables que determinan el desarrollo del proceso de adaptación en una nueva sociedad de acogida y para cada grupo inmigrante facilita enormemente la orientación de las políticas públicas de intervención en este ámbito para diferentes grupos inmigrantes.

Para ello, el trabajo persigue tres objetivos específicos. En primer lugar, analizar el efecto del origen de tres grupos inmigrantes sobre tres dimensiones específicas de aculturación (i.e., mantenimiento de la propia cultura de origen, 
adaptación cultural al nuevo contexto de acogida, y obligación de adaptación a la sociedad de acogida).

Los modelos clásicos de aculturación psicológica (p.e., Berry, 1997; Bourhis et al., 1997, 2010) estudian el proceso de aculturación en estrecha relación con la adaptación al nuevo contexto cultural. En todos los casos consideran que los inmigrantes deben plantearse dos cuestiones fundamentales cuando tienen que afrontar su proceso migratorio en el país de acogida. Por una parte, hasta qué punto es importante para el inmigrante mantener su identidad cultural o aspectos clave de su cultura de origen en este nuevo contexto ( $d i$ mensión de mantenimiento cultural); por otra, hasta qué punto valora y está dispuesto a adaptarse a aspectos clave de la sociedad de acogida (dimensión de adaptación cultural). Esta última dimensión ha recibido diferentes denominaciones según los autores y modelos (p.e., "contacto y participación en la sociedad de acogida", Berry, 1997; "adopción cultural”, Bourhis et al., 1997), pero en todos los casos alude al proceso de adquisición de y/o adaptación a aspectos clave del nuevo contexto cultural.

La perspectiva clásica en el estudio del proceso de aculturación ha tenido en cuenta la combinación de las respuestas de los inmigrantes a estas dos dimensiones para obtener las diferentes estrategias/actitudes u orientaciones de aculturación, es decir, las posibilidades con las que los inmigrantes pueden afrontar su proceso de aculturación y adaptación en su nuevo contexto cultural (i.e., integración, asimilación, separación y marginación). Sin embargo, algunos autores (p.e., Brown y Zagefka, 2011; Ryder, Alden y Paulhus, 2000; Ward y Rana-Deuba, 1999) defienden la importancia de diferenciar y medir ambas dimensiones (mantenimiento y adaptación cultural) por separado, tanto por razones teóricas como metodológicas. En este sentido, Brown y Zagefka (2011) afirman que medir las dimensiones subyacentes a las estrategias tradicionales de aculturación es esencial para determinar los efectos independientes de éstas, ya que con la aproximación tradicional sólo pueden analizarse los efectos combinados. Asimismo, es posible también que diferentes variables psicosociales influyan de forma distinta sobre cada una de estas dimensiones y esto sólo puede analizarse si se miden por separado. En este estudio mediremos, por tanto, la dimensión de mantenimiento de la cultura de origen, por un lado, y la dimensión de adaptación a la cultura de acogida, por otro.

Al mismo tiempo, introducimos una nueva dimensión de aculturación que ha sido menos considerada en la literatura: la obligación de adaptación a la cultura de acogida. Esta dimensión recoge la obligatoriedad o prescripción percibida por parte de los inmigrantes sobre el deber de adaptarse a la nueva cultura de acogida (entendiéndose ésta en íntima relación con la adopción de pautas culturales de la nueva sociedad de acogida).

El segundo objetivo del estudio es analizar el efecto del origen de los inmigrantes sobre otras variables psicosociales importantes, tradicionalmente estudiadas en la literatura sobre relaciones intergrupales. La mayoría de estas variables están también estrechamente relacionadas con los procesos generales y las dimensiones específicas de aculturación si bien no han sido estudiadas de forma simultánea en un mismo trabajo. Se trata de los estereotipos sobre el exogrupo (dimensiones de calidez y competencia) (Maisonneuve y Testé, 2007; Montreuil y Bourhis, 2001), la similitud intergrupal percibida, la identificación endogrupal y el enriquecimiento percibido (Piontkowski et al., 2000), la amenaza exogrupal percibida (Florack, Piontkowski, Rohmann, Balzer y Perzig, 2003), la naturaleza del contacto con el exogrupo o la percepción de la relación intergrupal. Por esta razón, el estudio pretende también (tercer objetivo) poner de manifiesto la capacidad predictiva de estas variables psicosociales y algunas variables sociodemográficas, consideradas de forma simultánea, sobre las dimensiones de mantenimiento y adaptación cultural de los inmigrantes.

La zona donde se ha realizado la investigación (Almería) se caracteriza por presentar una alta recepción de inmigrantes de diferentes orígenes. Así, de los 695.560 habitantes de la provincia de Almería, 150.865 (el 21.69\% de la población) son extranjeros que disponen de un certificado de registro o tarjeta de residencia. De entre todos los grupos inmigrantes que coexisten en la zona, los de origen marroquí (5.96\% de la población general), rumano (4.24\%) y ecuatoriano $(0.91 \%)$ son los más numerosos (Avance del Padrón a 1 de enero de 2010, INE, 2010). Además, se trata de grupos muy diferentes entre sí, ya que algunos hablan lenguas muy distintas a la del país de llegada (árabe, rumano) y otros la misma lengua. Asimismo, profesan diferentes religiones (musulmana, ortodoxa, católica), al tiempo que hay una gran disparidad entre las relaciones históricas (guerras, contenciosos, cooperación, etc.) que España ha mantenido con cada uno de estos grupos. Por ello, en general, esperamos que el origen de los inmigrantes establezca diferencias en las dimensiones de mantenimiento cultural, adaptación cultural y obligación de adaptación a la sociedad de acogida, y en las variables psicosociales estudiadas. Asimismo, esperamos que las variables que predicen cada una de esas dimensiones sean diferentes dependiendo del origen de los inmigrantes.

\section{Método}

\section{Participantes}

Participaron en la investigación 215 personas inmigrantes (71 marroquíes, 74 rumanos y 70 ecuatorianos). La edad de los participantes oscilaba entre 18 y 60 años, con una edad media en torno a 35 años (Grupo Marroquíes $=35.24$, DT = 9.25; Grupo Rumanos $=34.45, D T=10.38$; Grupo Ecuatorianos $=35.49, D T=10.13)$. Hombres y mujeres se distribuyen prácticamente a la mitad en todos los grupos, excepto en el caso del grupo de marroquíes, donde predominan los hombres $(67.6 \%)$, tal y como ocurre en la zona de investigación (65.7\% de hombres marroquíes vs. $34.3 \%$ de mujeres) y en España (60.3\% de hombres vs. $39.7 \%$ de mujeres) (Avance del Padrón a 1 de enero de 2010, INE, 2010). 
La mayor parte de la muestra de rumanos y ecuatorianos declara estar trabajando $(87.8 \%$ y $82.9 \%$ respectivamente), mientras que sólo el $57.7 \%$ de los marroquíes está en esta situación. Los marroquíes del estudio aseguran estar viviendo en nuestro país durante más años $(M=6.95, D T=7.56)$ que los rumanos $(M=4.71, D T=1.73)$ y los ecuatorianos $(M=$ $5.70, D T=1.88)$. Prácticamente la totalidad de la muestra manifiesta una situación documental regularizada $(91.2 \%$, $87.5 \%$ y $91.2 \%$ para marroquíes, rumanos y ecuatorianos, respectivamente).

\section{Diseño e Instrumentos}

Se trata de un estudio correlacional entregrupos, donde tres muestras de inmigrantes, concretamente de origen marroquí, rumano y ecuatoriano, respondieron a un cuestionario creado a tal efecto que incluía las siguientes variables:

Dimensiones de mantenimiento y adaptación cultural. Estas variables pretendían indagar tanto sobre el grado en que los participantes mantenían aspectos claves de su cultura de origen en nuestro país, como en qué medida creían que se habían adaptado a la cultura española en distintos aspectos, así como hasta qué punto creían que deberían hacerlo. Para medir las dos últimas variables de adaptación cultural y obligación percibida de adaptación se formularon dos preguntas que indagaban, respectivamente, sobre el grado de adaptación a la cultura española y sobre el grado en que deberían adaptarse a dicha cultura en distintos aspectos. Para medir la primera variable se preguntaba sobre el grado en que mantenian la cultura de origen en el país de acogida. En las tres preguntas las alternativas de respuesta oscilaban desde 1 (nada) hasta 5 (mucho). Los coeficientes alpha de Cronbach obtenidos para estas medidas fueron de .68 para la dimensión de mantenimiento cultural, de .71 para la dimensión de adaptación cultural, y de .81 para la obligación percibida de adaptación.

Estereotipo del exogrupo. Se midió a través de una traducción y adaptación de una medida de Fiske, Cuddy, Glick y Xu (2002, estudio 2) compuesta por 12 ítems: amistosos, bien intencionados, dignos de confianza, acogedores o afectuosos, buenas personas, sinceros, competentes, inteligentes, seguros de sí mismos, capaces, eficientes, y cualificados o bien preparados. Se pidió a los participantes que señalaran "en qué medida creían que cada una de esas características describia a las personas españolas", utilizando para ello una escala de respuesta de 5 puntos $(1=$ nada; $5=$ mucho $)$. El análisis factorial con rotación varimax $(\mathrm{KMO}=.89$; Prueba de esfericidad de Bartlett: $\left.\chi^{2}=1136.45, p<.001\right)$ realizado sobre esta escala reveló la existencia de dos factores diferentes con autovalores superiores a 1 que explicaban el 59.41\% de la varianza total. El primer factor, que explicó el 31.91\% de la varianza, estaba formado por seis características (dignos de confianza, amistosos, acogedores, bien intencionados, buenas personas y sinceros), constituyendo así la dimensión de Calidez (alpha de Cronbach de .89). El segundo factor explicó el $27.50 \%$ de la varianza y estaba formado por las seis características restantes (competentes, seguros de sí mismos, capaces, inteligentes, eficientes y bien preparados), conformando así la dimensión de Competencia (alpha de Cronbach de .82)

Amenaza exogrupal percibida. Esta variable pretende medir la amenaza percibida por parte del exogrupo en diferentes ámbitos de la vida (creencias y prácticas religiosas, valores y creencias en su comunidad, seguridad personal, y salud). Se utilizaron cuatro ítems de elaboración propia. A los participantes se les pide que indiquen "en qué medida sienten que, a causa de los españoles o debido a ellos, están en peligro una serie de cuestiones de su vida personal'. Para responder utilizaron una escala de 5 puntos $(1=$ nada; $5=$ mucho $)$. Se extrajo una puntuación media de la medida, cuya consistencia interna, medida a través del coeficiente alpha de Cronbach fue de .85 .

Identificación endogrupal. Esta variable se refiere al grado de identificación con el propio grupo nacional. Se midió con tres ítems, dos de ellos adaptados de Piontkowski et al. (2000), y utilizados en trabajos anteriores en nuestro país (Navas et al., 2004; Navas y Rojas, 2010). Concretamente, se trata de la intensidad de la identificación con el propio grupo (“¿Cuánto se identifica Vd. con la cultura del endogrupo nacional?"), la percepción de prototipicalidad endogrupal ("¿Cómo de parecido es $V d$. a una persona representativa de la cultura del endogrupo (el miembro promedio del endogrupo)?", y el sentimiento de pertenencia endogrupal ("¿En qué medida se siente $V d$. del endogrupo?”). En todos los casos los participantes respondían utilizando una escala que oscilaba desde 1 (nada) a 5 (mucho). La consistencia interna de esta medida fue de .75 (coeficiente alpha de Cronbach).

Similitud percibida entre endogrupo y exogrupo Se midió de forma general, utilizando un solo ítem ("En su opinión y en general, ¿cómo son las personas españolas en comparación con las de su endogrupo?") (Navas et al., 2004; Navas y Rojas, 2010). Las alternativas de respuesta oscilaban desde 1 (muy diferentes) hasta 5 (muy parecidas).

Naturaleza del contacto con el exogrupo. Como indicador de esta variable se empleó un único ítem ("¿Cómo considera Vd. que ha sido el contacto que ha tenido o tiene con personas españolas?') (Navas et al., 2004; Navas y Rojas, 2010), cuya escala de respuesta oscilaba desde 1 (muy desagradable) hasta 5 (muy agradable).

Percepción de la relación endo-exogrupo. Se midió también con un solo ítem de elaboración propia. Los participantes tenían que indicar "cómo creían que era la relación entre las personas de su endogrupo y las personas españolas". La escala de respuesta oscilaba desde 1 (muy conflictiva) a 5 (muy armoniosa).

Percepción de enriquecimiento por la inmigración. Esta variable se midió con tres ítems extraídos y adaptados del European Social Survey. Concretamente, se mide la percepción de enriquecimiento por la llegada de inmigrantes (sin especificar grupo de origen) en tres ámbitos del país receptor (economía, vida cultural, lugar para vivir). La escala de respuesta oscila en todos los casos entre 1 y 5 , siendo 1 la peor puntuación (p.e., peor economía por la llegada de inmigrantes) y 5 la 
mejor (p.e., el país es un lugar mejor para vivir por la llegada de inmigrantes).

Variables sociodemográficas. Se incluyeron las variables habituales en este tipo de investigación: sexo, edad, actividad principal, religión, grado de práctica religiosa, tiempo de estancia en España y situación documental en el momento de ser encuestados.

\section{Procedimiento}

Los cuestionarios fueron aplicados por tres encuestadores de la misma nacionalidad que los grupos encuestados (marroquí, rumana o ecuatoriana, según el caso) que en la mayoría de las ocasiones trabajaban y colaboraban con sindicatos y ONG de apoyo a inmigrantes (p.e., Red Acoge, Cruz Roja Española). Se les procuró una formación como entrevistadores y una tabla de cuotas siguiendo dos criterios: sexo de las personas a entrevistar y procedencia de las mismas marroquíes, rumanos o ecuatorianos-.

\section{Resultados}

\section{Análisis de datos}

Con el fin de comprobar si los diferentes grupos inmigrantes manifestaban diferencias en las tres dimensiones que indagaban sobre su proceso de aculturación en nuestro país, se llevó a cabo un MANOVA, siendo el grupo inmigrante la variable independiente, y el mantenimiento cultural, la adaptación cultural y la obligación de adaptación las variables dependientes. Para comprobar el efecto del origen del grupo inmigrante sobre las diferentes variables psicosociales, se realizaron diversos ANOVAs y un MANOVA, en el caso de los estereotipos. Finalmente, para comprobar qué variables (psicosociales y sociodemográficas) predecían mejor cada una de las dimensiones de aculturación para los diferentes grupos inmigrantes, se llevaron a cabo análisis de regresión múltiple por el método de pasos sucesivos para cada grupo inmigrante y para cada dimensión: mantenimiento cultural, adaptación cultural y obligación de adaptación.

Diferentes dimensiones de mantenimiento y adaptación cultural para diferentes grupos inmigrantes

El análisis reveló que existía un efecto del grupo inmigrante sobre las tres dimensiones de aculturación estudiadas.

Concretamente, el grupo inmigrante tuvo un efecto sobre la tendencia a mantener la cultura de origen, $F(2,194)=$ $12.48, p<.001, \eta^{2}=.11$. Los rumanos son los inmigrantes que mantienen significativamente menos costumbres de origen en comparación con los marroquíes $(p<.001)$ y con los ecuatorianos $(p=.003)$, no existiendo diferencias estadísticamente significativas entre estos dos últimos grupos en esta variable $(p=.553)$.

El grupo inmigrante también tuvo un efecto significativo sobre la tendencia a adaptarse a la sociedad de acogida, $F(2,194)$ $=14.05, p<.001, \eta^{2}=.13$. Los ecuatorianos son los inmigrantes que se adaptan significativamente más a la sociedad española, en comparación con los marroquíes $(p<.001)$, y con los rumanos $(p=.003)$. No se encontraron diferencias estadísticamente significativas en esta variable entre marroquíes y rumanos $(p=.124)$.

Finalmente, el grupo inmigrante también tuvo un efecto significativo sobre la obligación de adaptación, $F(2,194)=44.85$, $p=.001, \eta^{2}=.32$. En este caso eran los marroquíes los que menos consideran que deberían adaptarse a la cultura española, en comparación con rumanos $(p<.001)$ y ecuatorianos $(p<.001)$. No existían diferencias estadísticamente significativas entre estos dos grupos en esta variable $(p=.643)$.

\section{Diferentes percepciones en distintas variables psi- cosociales}

Tal como esperábamos, los análisis de varianza revelaron que los diferentes grupos inmigrantes mantenían percepciones endogrupales e intergrupales diferentes. En la Tabla 1 se muestran los estadísticos descriptivos de las diferentes variables.

Tabla 1. Estadísticos descriptivos de las variables del estudio para los tres grupos inmigrantes.

\begin{tabular}{|c|c|c|c|c|c|c|c|c|c|}
\hline & \multicolumn{3}{|c|}{ Marroquíes } & \multicolumn{3}{|c|}{ Rumanos } & \multicolumn{3}{|c|}{ Ecuatorianos } \\
\hline & $\overline{\mathrm{N}}$ & $M$ & $D T$ & $\overline{\mathrm{N}}$ & $M$ & DT & $\overline{\mathrm{N}}$ & $M$ & $D T$ \\
\hline Mantenimiento Cultura Origen & 70 & 4.11 & .54 & 74 & 3.58 & .44 & 62 & 4.06 & .88 \\
\hline Adaptación Cultura Acogida & 70 & 2.80 & .76 & 74 & 2.91 & .44 & 63 & 3.45 & 1.00 \\
\hline Obligación de Adaptación & 65 & 2.18 & .63 & 74 & 3.23 & .49 & 56 & 3.36 & 1.13 \\
\hline Estereotipos: Calidez & 71 & 3.58 & .54 & 74 & 3.84 & .65 & 70 & 3.48 & .80 \\
\hline Estereotipos: Competencia & 71 & 3.86 & .41 & 74 & 3.56 & .60 & 70 & 3.68 & .64 \\
\hline Amenaza exogrupal & 71 & 1.25 & .51 & 72 & 1.80 & .71 & 70 & 2.01 & .91 \\
\hline Identificación endogrupal & 71 & 4.22 & .50 & 74 & 3.37 & .58 & 70 & 4.34 & .54 \\
\hline Similitud endo-exogrupal & 71 & 3.34 & .84 & 74 & 3.45 & .74 & 70 & 2.93 & 1.31 \\
\hline Naturaleza del contacto & 68 & 3.57 & .72 & 72 & 4.04 & .66 & 69 & 3.83 & .94 \\
\hline Percepción relación endo-exogrupal & 71 & 3.55 & .60 & 74 & 3.92 & .72 & 70 & 3.64 & .93 \\
\hline Enriquecimiento percibido de la inmigración & 71 & 4.85 & .37 & 74 & 3.63 & .52 & 70 & 3.82 & .80 \\
\hline
\end{tabular}


Así, el grupo inmigrante tuvo un efecto sobre las percepciones estereotípicas que los inmigrantes mantienen sobre los españoles, tanto en la dimensión de calidez, $F(2,214)=5.35$, $p=.005, \eta^{2}=.05$, como en la de competencia, $F(2,214)=$ $5.25, p=.006, \eta^{2}=.05$. En el caso de la calider, los rumanos perciben a los españoles significativamente más cálidos de lo que los perciben los inmigrantes ecuatorianos $(p=.008)$. No existen diferencias estadísticamente significativas entre rumanos y marroquíes $(p=.07)$, ni entre marroquíes y ecuatorianos $(p=.707)$ en esta variable. En el caso de la competencia, los inmigrantes marroquíes perciben a los españoles significativamente más competentes de lo que los perciben los inmigrantes rumanos $(p=.006)$. Tampoco existen diferencias estadísticamente significativas entre marroquíes y ecuatorianos, $(p=.171)$, ni entre estos últimos y rumanos $(p=.426)$.

Con respecto a la amenaza exogrupal percibida se encontraron diferencias entre los grupos de inmigrantes, $F(2,212)=$ 20.25, $p<.001, \eta^{2}=.16$. Concretamente, los marroquíes se sienten menos amenazados por los españoles que los rumanos $(p<.001)$, y que los ecuatorianos $(p<.001)$. No existen diferencias estadísticamente significativas entre rumanos y ecuatorianos en esta variable $(p=.241)$.

Los análisis mostraron también que existían diferencias estadísticamente significativas entre los inmigrantes en su grado de identificación endogrupal, $F(2,214)=68.76, p<.001$, $\eta^{2}=.39$. Los rumanos eran los inmigrantes que menos se identificaban con su grupo en comparación con los marroquíes $(p<.001)$ y los ecuatorianos $(p<.001)$. Estos últimos se sentían fuertemente identificados con sus respectivos grupos, no estableciéndose diferencias estadísticamente significativas entre ambos $(p=.411)$.

Respecto a la naturaleza del contacto mantenido con autóctonos, también se encontraron diferencias estadísticamente significativas entre los inmigrantes, $F(2,208)=6.30, p=$ $.002, \eta^{2}=.06$. Los marroquíes perciben que el contacto con los españoles ha sido menos agradable de lo que perciben los rumanos $(p=.002)$. No existen diferencias estadísticamente significativas entre marroquíes y ecuatorianos $(p=.169)$, ni entre rumanos y ecuatorianos $(p=.263)$ en esta variable.

El grupo inmigrante tuvo también un efecto principal en la percepción de similitud endo-exogrupal, $F(2,214)=5.39, p=$ $.005, \eta^{2}=.05$. Contrariamente a lo esperado, los ecuatorianos son los que perciben menos similitud entre su grupo y los españoles, en comparación con los rumanos $(p=.008)$ y con los marroquíes $(p=.052)$. Sin embargo, no existen diferencias estadísticamente significativas entre estos dos grupos $(p=.807)$.

Se encontró un efecto principal del grupo inmigrante en la percepción de la relación endo-exogrupal, $F(2,214)=4.63, p=$ $.011, \eta^{2}=.04$. Los rumanos percibían significativamente mejor relación con los españoles que los marroquíes $(\phi=.015)$. No existían diferencias estadísticamente significativas en esta variable ni entre marroquíes y ecuatorianos $(p=.767)$, ni entre rumanos y ecuatorianos $(p=.097)$.

Finalmente, también se encontró un efecto principal del origen nacional sobre el enriquecimiento percibido que supone la inmigración, $F(2,214)=88.93, p<.001, \eta^{2}=.46$. Los inmigrantes marroquíes son el grupo que considera en mayor medida que la inmigración en general supone un enriquecimiento para el país receptor, en comparación con lo que perciben rumanos $(p<.001)$ y ecuatorianos $(p<.001)$, entre los cuales no existen diferencias en esta variable $(p=.172)$.

\section{Variables predictoras de las dimensiones de mante- nimiento y adaptación cultural}

El tercer objetivo del estudio era averiguar si las dimensiones de aculturación de los diferentes grupos inmigrantes (mantenimiento cultural, adaptación cultural y obligación de adaptación) podían predecirse a partir de variables distintas, tanto psicosociales (estereotipos, amenaza exogrupal percibida, identificación endogrupal, similitud endo-exogrupal, naturaleza del contacto, percepción de la relación endoexogrupal y enriquecimiento percibido), como sociodemográficas (tiempo de estancia). Con este objetivo se incluyeron todas las variables indicadas en un mismo bloque en el modelo de regresión múltiple y se analizaron sus efectos a través del método de pasos sucesivos para cada grupo inmigrante y para cada una de las dimensiones de aculturación.

Los resultados de los análisis de regresión lineal múltiple obtenidos para cada submuestra pueden verse en las Tablas 2,3 y 4 .

Tabla 2. Regresión lineal múltiple de las variables predictoras del mantenimiento de la cultura de origen para los tres grupos del estudio.

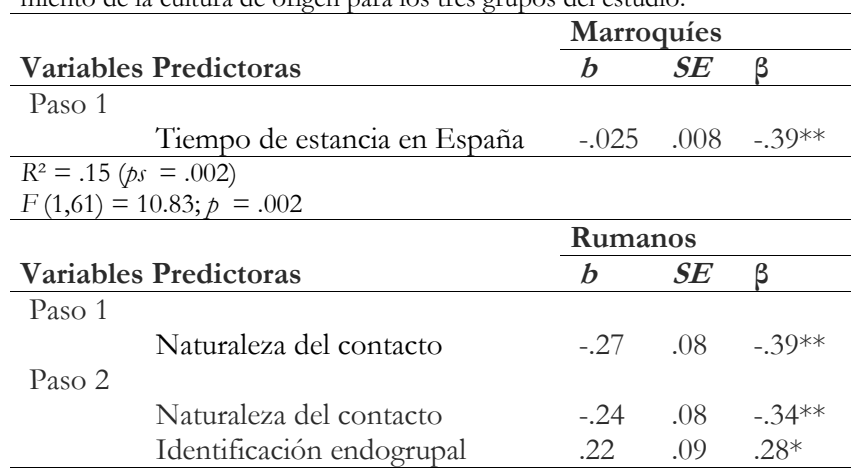

$\mathrm{R}^{2}=.15$ para Paso $1 ; \Delta \mathrm{R}^{2}=.08$ para Paso $2(p s=.013)$

$F(1,64)=11.12 ; p=.001$ para Paso 1

$F(2,64)=9.31 ; p<.001$ para Paso 2

\begin{tabular}{llll} 
& \multicolumn{3}{l}{ Ecuatorianos } \\
\cline { 2 - 3 } Variables Predictoras & $b$ & $S E$ & $\beta$ \\
\hline
\end{tabular}

Paso 1

Percepción Relación

endo-exogrupal $\begin{array}{lll}46 & .11 \quad .53 * * *\end{array}$

$\mathrm{R}^{2}=.28(p s<.001)$

$F(1,48)=18.30 ; p<.001$

Nota. $b$ representa el coeficiente de regresión no estandarizado. ${ }^{*} p<.05,{ }^{* *} p$ $<.01, * * * p<.001$.

En relación con el mantenimiento de la cultura de origen (ver Tabla 2), los resultados para la submuestra de inmigrantes marroquíes mostraron que el tiempo de estancia era la variable con mayor poder predictivo, de forma que mantenían menos costumbres de origen a medida que llevaban más 
años residiendo en España y a la inversa (menos tiempo de estancia, más mantenimiento de costumbres de origen). Para la muestra de inmigrantes rumanos, en cambio, el modelo formado por la naturaleza del contacto y la identificación endogrupal es el mejor predictor de esta dimensión. Es decir, un contacto desagradable con miembros del exogrupo y una alta identificación con el endogrupo predicen una mayor tendencia a mantener más costumbres de la cultura de origen (y a la inversa). En cuanto a los ecuatorianos, la variable que mejor predijo el mantenimiento de su cultura de origen fue la percepción de la relación intergrupal entre ecuatorianos y españoles: a medida que los ecuatorianos perciben que las relaciones entre españoles y ecuatorianos son más armoniosas, tienden a mantener más costumbres de origen, y a la inversa (relaciones intergrupales conflictivas, menos mantenimiento de costumbres de origen).

Tabla 3. Regresión lineal múltiple de las variables predictoras de la adaptación a la cultura de acogida para los tres grupos del estudio

\begin{tabular}{|c|c|c|c|}
\hline \multirow{2}{*}{ Variables Predictoras } & \multicolumn{3}{|c|}{ Marroquíes } \\
\hline & $b$ & $S E$ & $\beta$ \\
\hline \multicolumn{4}{|l|}{ Paso 1} \\
\hline Identificación endogrupal & -.72 & .17 & $-.47 * * *$ \\
\hline \multicolumn{4}{|l|}{ Paso 2} \\
\hline Identificación endogrupal & -.58 & .16 & $-.38 * *$ \\
\hline Tiempo de estancia en España & .04 & .01 & $.37 * *$ \\
\hline \multicolumn{4}{|c|}{$\begin{array}{l}\mathrm{R}^{2}=.22 \text { para Paso } 1 ; \Delta R^{2}=.13 \text { para Paso } 2(p s=.001) \\
F(1,61)=17.46 ; p=.001 \text { para Paso } 1 \\
F(2,61)=15.96 ; p<.001 \text { para Paso } 2\end{array}$} \\
\hline \multirow[b]{2}{*}{ Variables Predictoras } & \multicolumn{3}{|c|}{ Rumanos } \\
\hline & $b$ & $S E$ & $\beta$ \\
\hline \multicolumn{4}{|l|}{ Paso 1} \\
\hline Calidez (estereotipo) & .33 & .08 & $.45 * * *$ \\
\hline \multicolumn{4}{|l|}{$\begin{array}{l}R^{2}=.21(p s=.001) \\
F(1,64)=16.40 ; p<.001\end{array}$} \\
\hline \multirow[b]{2}{*}{ Variables Predictoras } & \multicolumn{3}{|c|}{ Ecuatorianos } \\
\hline & $b$ & $S E$ & $\beta$ \\
\hline \multicolumn{4}{|l|}{ Paso 1} \\
\hline Percepción Similitud intergrupal & .55 & .07 & $.74 * * *$ \\
\hline Percepción Similitud intergrupal & .47 & .07 & $.63 * * *$ \\
\hline Enriquecimiento percibido & .34 & .12 & $.28^{* *}$ \\
\hline $\begin{array}{l}F(1,49)=56.81 ; p<.001 \text { para Paso } 1 \\
F(2,49)=36.74 ; p<.001 \text { para Paso } 2\end{array}$ & $.006)$ & & \\
\hline $\begin{array}{l}\text { Nota. } b \text { representa el coeficiente de regresión no } \\
<.01, * * *<<.001 .\end{array}$ & & & \\
\hline
\end{tabular}

En cuanto a las variables predictoras de la dimensión de adaptación a la sociedad de acogida, los análisis mostraron que, en el caso de los marroquíes, el modelo formado por la identificación endogrupal y el tiempo de estancia era el que presentaba más poder predictivo. Es decir, los marroquíes más identificados con su endogrupo y que llevan poco tiempo residiendo en España se adaptan menos a la cultura española y a la inversa (menos identificación y más años en España, más adaptación). Para los inmigrantes rumanos, sin embargo, la calidez con la que son percibidos los españoles es la variable que mejor predice su tendencia adaptarse a la cultura de acogida: tienden a adaptarse más a la cultura española a medida que perciben a los españoles como personas más cálidas (dignas de confianza, sinceras o buenas personas). Finalmente, el modelo formado por la similitud endoexogrupal y la percepción de enriquecimiento por la inmigración es el mejor predictor de la adaptación de los ecuatorianos. Concretamente, a medida que los ecuatorianos perciben a los españoles como más similares a ellos y que la inmigración supone un mayor enriquecimiento para el país receptor, se adaptan más a la cultura de acogida (véase Tabla 3).

En relación a la obligación de adaptación, ninguna de las variables del estudio la predice en los inmigrantes de origen marroquí. Para los inmigrantes rumanos, de nuevo la calidez es la variable que mejor predice esta dimensión. Es decir, a medida que perciben a los españoles como más cálidos, consideran que deberían adaptarse más. Finalmente, para los ecuatorianos el modelo que mejor predice esta dimensión está formado por la percepción de similitud intergrupal, la naturaleza del contacto mantenido con miembros del exogrupo y la percepción de enriquecimiento por la inmigración. En este sentido, a medida que el contacto con miembros del exogrupo ha sido más agradable, se perciben como más similares a los españoles y perciben que los inmigrantes suponen un mayor enriquecimiento para el país de acogida, piensan que deberían adaptarse más a la cultura española (véase Tabla 4).

Tabla 4. Regresión lineal múltiple de las variables predictoras de la obligación de adaptación para los tres grupos del estudio

\begin{tabular}{|c|c|c|c|c|}
\hline \multirow{2}{*}{\multicolumn{2}{|c|}{ Variables Predictoras }} & \multicolumn{3}{|c|}{ Rumanos } \\
\hline & & $b$ & $S E$ & $\beta$ \\
\hline \multicolumn{5}{|c|}{ Paso 1} \\
\hline & Calidez (estereotipo) & .39 & .09 & $.47 * * *$ \\
\hline \multicolumn{5}{|c|}{$\begin{array}{l}R^{2}=.22(p s<.001) \\
F(1,64)=17.98, p<.001\end{array}$} \\
\hline \multirow{2}{*}{\multicolumn{2}{|c|}{ Variables Predictoras }} & \multicolumn{3}{|c|}{ Ecuatorianos } \\
\hline & & $b$ & $S E$ & $\beta$ \\
\hline \multicolumn{5}{|c|}{ Paso 1} \\
\hline & $\begin{array}{l}\text { Percepción Similitud } \\
\text { intergrupal }\end{array}$ & .55 & .11 & $.61 * * *$ \\
\hline \multicolumn{5}{|l|}{ Paso 2} \\
\hline & $\begin{array}{l}\text { Percepción Similitud } \\
\text { intergrupal }\end{array}$ & .43 & .11 & $.48^{* * *}$ \\
\hline & Naturaleza del contacto & .45 & .16 & $.34 * *$ \\
\hline \multicolumn{5}{|l|}{ Paso 3} \\
\hline & $\begin{array}{l}\text { Percepción Similitud } \\
\text { intergrupal }\end{array}$ & .36 & .11 & $.40 * *$ \\
\hline & Naturaleza del contacto & .47 & .15 & $.35^{* *}$ \\
\hline & Enriquecimiento percibido & .32 & .14 & $.25^{*}$ \\
\hline
\end{tabular}

$R^{2}=.38$ para Paso $1 ; \Delta R^{2}=.10$ para Paso $2 ; \Delta R^{2}=.06$ para Paso $3(p s=$ $.034)$

$F(1,43)=25.34 ; p<.001$ para Paso 1

$F(2,43)=18.64 ; p<.001$ para Paso 2

$F(3,43)=15.21 ; p<.001$ para Paso 3

Nota. $b$ representa el coeficiente de regresión no estandarizado. ${ }^{*} p<.05,{ }^{* *} p$ $<.01, * * * p<.001$. 


\section{Discusión}

El objetivo fundamental del presente estudio era comprobar la influencia del origen nacional de tres grupos inmigrantes (marroquíes, rumanos y ecuatorianos) tanto sobre las dimensiones de mantenimiento y adaptación cultural, como sobre otras variables psicosociales estrechamente relacionadas con ellas, así como determinar las variables que mejor predicen estas dimensiones en cada grupo inmigrante. La finalidad última de nuestro trabajo era tratar de profundizar en la comprensión de los factores que influyen en cómo los inmigrantes afrontan su proceso migratorio en el país de acogida, así como resaltar la especificidad de este proceso para diferentes grupos inmigrantes en función de su procedencia.

De acuerdo con nuestras predicciones, los resultados obtenidos evidencian que efectivamente el origen de los inmigrantes tiene un efecto sobre las dimensiones de aculturación estudiadas. Así, los rumanos del estudio son los inmigrantes que mantienen menos costumbres de origen, los ecuatorianos los que más se adaptan a la sociedad de acogida, y los marroquíes los que menos consideran que deberían adaptarse a la cultura española. Estas diferencias encontradas son coherentes con los planteamientos defendidos en algunos modelos de aculturación psicológica en los que el origen de los inmigrantes se muestra como una variable muy importante en el proceso de aculturación (p.e., Bourhis et al., 1997; Navas et al., 2004; Navas y Rojas, 2010; Piontkowski et al., 2000). A la vez, los resultados están indicando que este proceso es sumamente complejo y diverso, hasta tal punto que cada grupo inmigrante, por diferentes razones relacionadas con su origen, lo experimenta y lo afronta de forma diferente.

En relación con el segundo objetivo, los resultados de nuestro estudio también confirman la idea de que diferentes grupos inmigrantes difieren en sus percepciones del exogrupo nacional y de la realidad intergrupal, percepciones estrechamente relacionadas con su proceso de afrontamiento de la experiencia migratoria en un nuevo contexto cultural.

Así, los inmigrantes de origen marroquí, en comparación con los rumanos, son los que perciben a los españoles como más competentes (estereotipo) y creen que el contacto con españoles ha sido menos agradable. Además, son los que se sienten menos amenazados por parte de los españoles y los que más consideran que los inmigrantes suponen un enriquecimiento para el país de acogida, en comparación con los otros dos grupos. Por su parte, los inmigrantes rumanos perciben a los españoles como más cálidos (en comparación con ecuatorianos), consideran que la relación intergrupal es más armoniosa (en comparación con marroquíes), y son los que menos se identifican con su propio grupo (en comparación con los otros dos grupos inmigrantes). Finalmente, los inmigrantes ecuatorianos, contrariamente a lo esperado, son los que perciben menos similitud entre su grupo y los españoles, en comparación con los otros dos grupos. Este último resultado posiblemente pueda explicarse por la necesidad de conseguir distintividad óptima (Brewer, 2001). En este caso, los ecuatorianos, un grupo que comparte lengua y religión con la mayoría española, se percibiría más diferente a los españoles de lo que lo hacen marroquíes y rumanos, como una forma de diferenciarse positivamente de un exogrupo muy parecido al propio endogrupo.

Por otra parte, los resultados del estudio confirman la idea de que el patrón de variables que subyace a las diferentes dimensiones de aculturación utilizadas en este trabajo varía según el grupo considerado (Piontkowski et al., 2000). En el caso de los inmigrantes marroquíes, el tiempo de estancia en la sociedad de acogida y la identificación endogrupal son las dos variables que predicen sus dimensiones de aculturación (i.e., mantenimiento y adaptación cultural). No obstante, ninguna variable predijo la obligación a adaptarse a la cultura de acogida, probablemente porque este grupo de inmigrantes es el que considera en menor medida que deben adaptarse a la cultura española.

En el caso de los inmigrantes rumanos, la identificación endogrupal también es predictora, junto con la naturaleza del contacto con españoles, de la dimensión de mantenimiento cultural. En este sentido, no extraña, por tanto, que los rumanos sean los inmigrantes que significativamente mantienen menos costumbres de origen, ya que también son los que se identifican menos con su grupo y manifiestan tener un contacto agradable con españoles. Sin embargo, en la adaptación a la sociedad de acogida y en la obligación de adaptarse de los rumanos tiene más peso una dimensión del estereotipo exogrupal: la calidez con la que son percibidos los españoles. Así, los rumanos tienden a adaptarse más a la sociedad de acogida y creen que deberían adaptarse más a medida que perciben a los españoles como personas más cálidas (dignas de confianza, sinceras o buenas personas). La naturaleza del contacto también desempeña un papel relevante para los rumanos en esta última dimensión: creen que deberían adaptarse más a la sociedad de acogida a medida que el contacto con españoles ha sido más agradable.

Finalmente, en el caso de los inmigrantes ecuatorianos las variables que predicen sus dimensiones de aculturación son cuatro y diferentes a las de los grupos anteriores. Así, la percepción de la relación endo-exogrupal predice en positivo el mantenimiento de costumbres de origen de este grupo. Por su parte, la percepción de similitud endo-exogrupal junto con la percepción de enriquecimiento por la inmigración es el modelo que mejor predice la adaptación a la cultura de acogida. Estas dos variables, junto con la naturaleza del contacto exogrupal, predicen en positivo la obligación de adaptarse a la sociedad de acogida. Resulta interesante que la similitud endo-exogrupal sea una variable predictora importante en dos dimensiones de aculturación en este grupo inmigrante y no aparezca en el resto de los grupos. Por las razones mencionadas en la introducción, el grupo inmigrante del que aparentemente cabría esperar más proximidad o cercanía a la sociedad de acogida (y, por tanto, mayor similitud endo-exogrupal percibida) en diferentes dimensiones (p.e., lengua, religión) sería el procedente de Ecuador (en comparación con el de Marruecos o Rumanía). Sin embargo, nues- 
tros resultados muestran justo lo contrario: son precisamente los inmigrantes ecuatorianos (en comparación con rumanos y marroquíes) los que se perciben menos similares a los españoles. Estos resultados coinciden con los encontrados en otros estudios realizados en nuestro país (Navas y Rojas, 2010) y parecen darse en las dos direcciones: inmigrantes ecuatorianos y autóctonos que opinan sobre ellos. Ambos grupos perciben una baja similitud intergrupal. Quizá la necesidad de diferenciarse en positivo a la que ya hemos aludido anteriormente (Brewer, 2001) pueda explicar también este resultado.

Es importante destacar también el papel que tiene la dimensión estereotípica de calidez a la hora de predecir la adaptación a la cultura de acogida y la obligación a adaptarse en los inmigrantes rumanos, que son los que perciben como más cálidos a los españoles (en comparación con los ecuatorianos). La inclusión de las dimensiones de los estereotipos mantenidos por los inmigrantes sobre los autóctonos en nuestro estudio permite comprobar un resultado que principalmente se había obtenido en grupos mayoritarios hasta el momento (p.e., Maisonneuve y Testé, 2007; Montreuil y Bourhis, 2001). Concretamente, que los estereotipos que se mantienen sobre los exogrupos (autóctonos o inmigrantes) influyen sobre las dimensiones de aculturación de ambos grupos. Así, nuestro estudio muestra que percibir a los autóctonos como cálidos predice en los inmigrantes rumanos tanto su adaptación a la sociedad española como su obligación de adaptarse. Posiblemente, esto se deba a que la calidez es resultado de la percepción de la interdependencia mutua entre endo y exogrupo, y de la valoración de las intenciones de los otros hacia nosotros (Fiske et al., 2002) y, por ello, tenga tanta influencia en las relaciones intergrupales. La competencia, sin embargo, otra de las dimensiones del Modelo del Contenido de los Estereotipos (Fiske et al., 2002), que no aparece como predictora en ningún grupo de nuestro estudio, deriva del estatus social del exogrupo, y no está relacionada, por tanto, con la intenciones de los otros hacia nosotros.

Junto a la calidez, la percepción de armonía intergrupal y la naturaleza del contacto también desempeñan un papel importante en las dimensiones de aculturación de rumanos y ecuatorianos. Estos resultados podrían estar indicando que las dimensiones de mantenimiento y adaptación cultural de los inmigrantes pueden estar mediadas por el grado en el que éstos se sienten aceptados o rechazados (Bourhis y Gagnon, 1994; Moghaddam y Taylor, 1987) a través del contacto mantenido con miembros del grupo mayoritario o de la percepción de la relación intergrupal en general.

\section{Conclusiones}

En definitiva, nuestros resultados indican claramente cuáles son las variables sobre las que habría que intervenir para que el proceso de aculturación de los inmigrantes se produzca en las mejores condiciones posibles. Y tal y como pronosticábamos al comienzo de nuestro trabajo, estas variables son distintas dependiendo de la procedencia de los inmigrantes, mostrando la especificidad de este proceso para diferentes grupos inmigrantes.

Como destacan Choi y Thomas (2009), es esencial que los responsables en el ámbito de la formulación de políticas públicas y en las profesiones asistenciales comprendan las actitudes de aculturación y las percepciones de diferentes grupos inmigrantes, ya que no todos los inmigrantes tienen la misma forma de afrontar estos procesos en su nuevo contexto cultural. En este sentido, se recomendaría que se analizasen las variables que parecen tener más peso en las dimensiones de mantenimiento y adaptación cultural expresadas por cada grupo inmigrante, con el fin de elaborar programas de intervención específicos para cada uno de ellos que faciliten su proceso de adaptación al nuevo contexto cultural.

Nuestro estudio pone de manifiesto, además, que las variables que influyen en cada dimensión de aculturación son, en la mayoría de las ocasiones, diferentes. En este sentido, los responsables de la creación y desarrollo de políticas públicas de inmigración e integración deberían tener en cuenta que los aspectos sobre los que hay que intervenir para fomentar bien el mantenimiento de la propia cultura de los inmigrantes, bien la adopción de la nueva cultura de acogida, son y deberían ser diferentes.

La comprensión de la especificidad del proceso de aculturación, tomando en consideración tanto el origen del grupo inmigrante involucrado como las dimensiones concretas analizadas, debería convertirse en el principio de actuación que guiara el diseño de las políticas migratorias, en aras de alcanzar una convivencia intergrupal no conflictiva en nuestro país.

Finalmente, es importante reconocer que el estudio que presentamos cuenta con algunas limitaciones. Principalmente, al tratarse de un estudio correlacional no se pueden establecer relaciones causales ni confirmar la direccionalidad de los efectos encontrados. Posteriores estudios de carácter experimental deberán llevarse a cabo para poder constatar el papel determinante de ciertas variables en los procesos de aculturación de diferentes grupos inmigrantes. Por otra parte, las muestras de inmigrantes del estudio no son suficientemente numerosas ni representativas, ya que han sido seleccionadas de forma incidental y su tamaño no es grande.

No obstante, a pesar de las limitaciones, el presente estudio nos permite comprender cómo el origen de los inmigrantes influye de forma esencial en las dimensiones de mantenimiento y adaptación cultural y en las percepciones intergrupales. En definitiva, como indican Piontkowski et al. (2000), los diferentes patrones de variables predictoras reflejan la diversidad cultural de los grupos inmigrantes, en nuestro caso de marroquíes, rumanos y ecuatorianos, y esto puede ayudarnos a comprender las dificultades que las personas de diferentes grupos culturales experimentan cuando tienen que adaptarse a una nueva sociedad de acogida.

Agradecimientos.- Esta investigación ha sido desarrollada dentro de un Proyecto de Excelencia financiado por la Junta de Andalucía 
(Consejería de Innovación y Ciencia; Referencia P09-SEJ-4657) y fondos FEDER; de un proyecto de investigación financiado por la Secretaría de Estado para Universidades, Investigación y Desarrollo

\section{Referencias}

Berry, J. W. (1997). Immigration, acculturation and adaptation. Applied Psychology, 46, 5-68.

Bourhis, R. Y., Moïse, L. C., Perreault, S. y Senécal, S. (1997). Towards an interactive acculturation model: A social psychological approach. International Journal of Psychology, 32, 369-386. doi: 10.1080/002075997400629

Bourhis, R. Y., Barrette, G. El-Geledi, S. y Schmidt, R. (2009). Acculturation orientations and social relations between immigrant and host community members in California. Journal of Cross-Cultural Psychology, 40, 443-467. doi: $10.1177 / 0022022108330988$

Bourhis, R. Y. y Gagnon, A. (1994). Préjugés, discrimination et relations intergroupes. En R. J. Vallerand (Ed.), Les fondements de la Psychologie Sociale (pp. 707-773). Boucherville, Québec: Gaetan Morin.

Bourhis, R. Y., Montaruli, E., El-Geledi, S., Harvey, S.P., Barrette, G. (2010). Acculturation in multiple host community settings. Journal of Social Issues, 66, 780-802.

Brewer, M. B. (2001). In-group identification and intergroup conflict: When does in-group love become out-group hate? En R. Ashmore y L. Jussim (Eds.), Social identity, intergroup conflict, and conflict reduction (pp. 17-41). New York: Oxford University Press.

Brown, R. y Zagefka, H. (2011). The Dynamics of Acculturation: An Intergroup Perspective. En J.M. Olson y M.P. Zanna (Eds.), Advances in Experimental Social Psychology (Vol. 44, pp. 129-184). Burlington: Academic Press.

Buriel, R. (1993). Childrearing orientations in Mexican American families: The influence of generation and sociocultural factors. Journal of Marriage and the Family, 55, 987-1000. doi: 10.2307/352778

Choi, J. y Thomas, M. (2009). Predictive factors of acculturation attitudes and social support among Asian immigrants in the USA. International Journal of Social Welfare, 18, 76-84. doi: 10.1111/j.14682397.2008.00567.x

Cuddy, A. J., Fiske, S. T. y Glick P. (2008). Warmth and Competence as Universal Dimensions of Social Perception: The Stereotype Content Model and the BIAS Map. En M. P. Zanna (Ed.), Advances in Experimental Social Psychology (pp. 61-149). New York: Academic Press.

Fiske, S. T., Cuddy, A. J., Glick, P. y Xu, J. (2002). A model of (often mixed) stereotype content: Competence and warmth respectively follow from perceived status and competition. Journal of Personality and Social Psychology, 82, 878-902. doi: 10.1037//0022-3514.82.6.878

Fiske, S. T., Xu, J., Cuddy, A. C. y Glick, P. (1999). (Dis)respecting versus (dis)liking: Status and interdependence predict ambivalent stereotypes of competence and warmth. Journal of Social Issues, 55, 473-491. doi: 10.1111/0022-4537.00128

Florack, A., Piontkowski, U., Rohmann, A., Balser, J. y Perzing, S. (2003). Perceived intergroup threat and attitudes of host community members towards immigrant acculturation. Journal of Social Psychology, 143, 633648.

Instituto Nacional de Estadística (2010). Avance del Padrón a 1 de enero de 2010. Obtenido del Instituto Nacional de Estadística en:

http: / / wnw.ine.es $/$ jaxi $/$ menu.do?type $=$ pcaxis ófile $=$ pcaxis ópath $=\% 2 \mathrm{Ft} 20 \% 2$ $\mathrm{Fe} 245 \% 2 \mathrm{Fp} 04 \% 2 \mathrm{~F} \% 2 \mathrm{Fa} 2010$ dentro del Plan Nacional I+D (Referencia SEJ2004-07369/PSIC), y del Programa FPU del Ministerio de Educación y Ciencia (Referencia AP2009-4881).

Lee, T. L. y Fiske, S. T. (2006). Not an out group, not yet an in group: Immigrants in the Stereotype Content Model. International Journal of Intercultural Relations, 30, 751-768. doi: 10.1016/j.ijintrel.2006.06.005

Mendoza, R. H. (1989). An empirical scale to measure type and degree of acculturation in Mexican American adolescents and adults. Journal of Cross-Cultural Psychology, 20, 372-385. doi: 10.1177/0022022189204003

Maisonneuve, Ch. y Testé, B. (2007). Acculturation preferences of a host community: The effects of immigrant acculturation strategies on evaluations and impression formation. International Journal of Intercultural Relations, 31, 669-688. doi:10.1016/j.ijintrel.2007.06.001

Moghaddam, F. M. y Taylor, D. M. (1987). The meaning of multiculturalism for visible minority immigrant women. Canadian Journal of Behavioural Science, 19, 121-136.

Montreuil, A. y Bourhis, R. Y. (2001). Majority acculturation orientations toward valued and devalued immigrants. Journal of Cross-Cultural Psychology, 32, 698-719. doi: 10.1177/0022022101032006004

Navas, M. S., Pumares, P., Sánchez, J., García, M. C., Rojas, A. J., Cuadrado, I., Asensio, M. y Fernández, J. S. (2004). Estrategias y actitudes de aculturación: la perspectiva de los inmigrantes y de los autóctonos en Almería. Sevilla: Dirección General de Coordinación de Políticas Migratorias. Consejería de Gobernación (Junta de Andalucía).

Navas, M. S. y Rojas, A. J. (Coords.) (2010). Aplicación del Modelo Ampliado de Aculturación relativa (MAAR) a nuevos colectivos de inmigrantes en Andalucia: rumanos y ecuatorianos. Sevilla: Consejería de Empleo (Junta de Andalucia).

Piontkowski, U., Florack, A., Hölker, P. y Obdrzálek, P. (2000). Predicting acculturation attitudes of dominant and non-dominant groups. International Journal of Intercultural Relations, 24, 1-26. doi:10.1016/S01471767(99)00020-6

Redfield, R., Linton, R. y Herskovits, M. (1936). Memorandum on the study of acculturation. American Anthropologist, 38, 149-152.

Rohmann, A., Florack, A. y Piontkowski, U. (2006). The role of discordant acculturation attitudes in perceived threat: An analysis of host and immigrant attitudes in Germany. International Journal of Intercultural Relations, 30, 683-702. doi: 10.1016/j.ijintrel.2006.06.006

Ryder, A. G., Alden, L. E., y Paulhus, D. L. (2000). Is acculturation unidimensional or bidimensional? A head-to-head comparison in the prediction of personality, self-identity, and adjustment. Journal of Personality and Social Psychology, 79, 49-65.

Schmitz, P. G. (1987). Acculturation attitudes and beliefs of immigrants. Psychology \& Developing Societies, 4, 117-131.

Ward, C. y Rana-Deuba, A. (1999). Acculturation and adaptation revisited. Journal of Cross-Cultural Psychology, 30, 422-442.

Worchel, S. (2004). Algunos comentarios sobre inmigración y aculturación. En M. S. Navas et al., Estrategias y actitudes de aculturación: la perspectiva de los inmigrantes y de los autóctonos en Almería (pp. 15-19). Sevilla: Dirección General de Coordinación de Políticas Migratorias. Consejería de Gobernación (Junta de Andalucía).

(Artículo recibido: 24-05-2011, revisión: 28-11-2011, aceptado: 07-12-2011) 